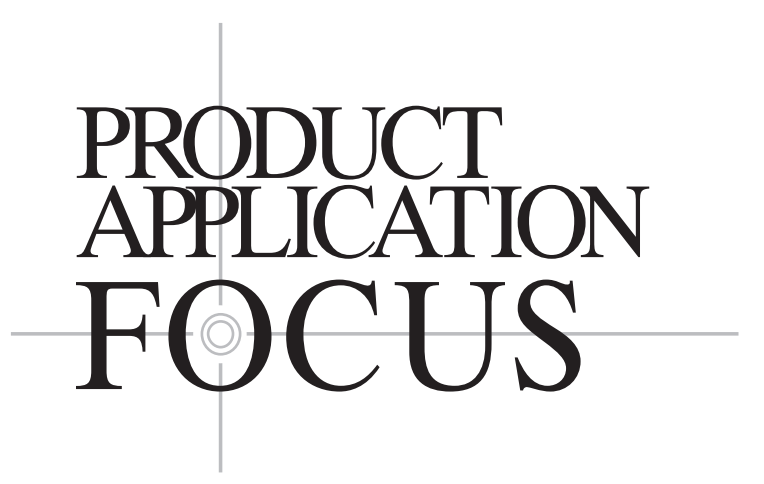

\title{
Development of a Chemiluminescence-Based Ribonuclease Protection Assay
}

\author{
Christopher Rosenau, Barbara Kaboord, and M. Walid Qoronfleh \\ Pierce Milwaukee, LLC, Milwaukee, WI, USA
}

BioTechniques 33:1354-1358 (December 2002)

\begin{abstract}
The ribonuclease protection assay (RPA) is a widely used method for the detection and quantification of specific mRNA transcripts in a complex mixture of total RNA or mRNA molecules. While exhibiting many advantages over other RNA detection methods, RPAs are traditionally performed using radiolabeled probes that often require gel purification steps and lengthy exposure times to visualize results. Moreover, these probes can only be used for 1-2 weeks because of their short isotopic half-life and radiolysis. We report a method that improves the traditional RPA by replacing radiolabeled probes with biotinylated probes and lengthy exposure times with quick, streptavidin/HRP-based chemiluminescent detection technology. Biotinylated probes can be used without gel purification and are stable for years, as opposed to weeks. Most importantly, our streptavidin/HRP-based chemiluminescent technology enables us to achieve sensitivity results similar to radioactive RPAs and to detect multiple transcripts in a single sample more efficiently. Furthermore, this new protocol addresses and eliminates the one major drawback unique to using biotinylated probes in chemiluminescent RPAs: a confounding artifact, not seen when running radioactive RPAs but commonly detected when using certain biotinylated rare message probes.
\end{abstract}

\section{INTRODUCTION}

The mechanisms that regulate gene expression and protein activity are complex. Frequently, changes in cellular mRNA levels directly correlate to changes in their corresponding protein levels. Although there are exceptions to this rule (1), monitoring mRNA levels in response to genetic manipulations or small-molecule stimulation is the approach favored by scientists studying gene expression. The ribonuclease protection assay (RPA) is a sensitive method to detect and quantify specific mRNA transcripts in a complex mixture of total RNA or
mRNA molecules (8). RPAs offer distinct advantages over other RNA detection methods. In an RPA reaction, the probe and target mRNA are hybridized in solution where target availability is maximal, affording increased detection of rare messages $(2,5)$. In addition, RPAs enable the researcher to probe for multiple transcripts in one sample of RNA. This "multiplexing" technique is not suited for quantitative RT-PCR, which rivals RPA sensitivity, but also commonly requires extensive optimization. Northern blots, which are 10 times less sensitive that RPAs (2), can be probed for multiple transcripts but require multiple stripping and re-probing of a single blot (4).

The advantages that RPAs exhibit over these RNA detection methods are often overshadowed, however, by the fact that RPAs have historically used radiolabeled probes. The disadvantages of the radioactive RPA technique are 2-fold. First, the researcher must synthesize and purify new radioactive probes weekly to guard against short isotope half-life as well as radiolysis. RPA results can only be as good as the probe used to generate them. Second, radioactive probes require lengthy exposure times, often overnight, to visualize results. Since RPAs frequently need to be optimized for the researcher's specific target RNA and probe, lengthy exposure times common to radioactive RPAs significantly increase the amount of time necessary to generate valid RPA results.

Our new RPA method replaces the traditional 32P-labeled RPA probes with biotinylated probes that can be detected using streptavidin/HRP chemiluminescent reagents. This method improves upon homemade chemiluminescent RPAs (6) by combining RNase inactivation and precipitation steps to increase efficiency, and by utilizing enhanced detection reagents to increase sensitivity. In our assay, a biotinylated RNA probe complementary for the sequence of interest hybridizes in solution to that target mRNA sequence. The resulting duplex is then digested by RNases specific for ssRNA, leaving only the desired fragment that has been "protected" as part of the double-strand- 
ed probe/mRNA duplex. The digest is subjected to electrophoresis on a denaturing polyacrylamide gel and transferred to a nylon membrane. The biotinylated probes are then detected and quantified using a streptavidin-HRP conjugate and chemiluminescent substrate, followed by a very brief (minutes) exposure to X-ray film or a CCD camera.

Here we describe the advantages our new chemiluminescent RPA affords researchers over common mRNA detection methods such as Northern blotting and quantitative RT-PCR, as well as over traditional radioactive RPA methods. This new chemiluminescent RPA alternative yields fast, sensitive, and quantitative results while maintaining the ability to detect multiple transcripts in one complex total RNA sample.

\section{MATERIALS AND METHODS}

\section{Preparation of Radioactive Probes}

Radioactive probes of high specific activity were prepared by in vitro transcription reactions using T7 MAXIscript ${ }^{\mathrm{TM}}$ Transcription kits (Ambion, Austin, TX, USA) in which CTP was completely replaced with $[\alpha-32 \mathrm{P}] \mathrm{CTP}(400 \mathrm{Ci} / \mathrm{mmol}, 10$ $\mathrm{mCi} / \mathrm{mL}$; Amersham Biosciences, Piscataway, NJ, USA), and Ambion Antisense DNA templates per the manufacturer's instructions. The in vitro transcription reaction was incubated at $37^{\circ} \mathrm{C}$ for $30 \mathrm{~min}$, and the excess original template digested with $2 \mathrm{U}$ DNase I for $15 \mathrm{~min}$ at $37^{\circ} \mathrm{C}$. Adding EDTA to a concentration of $0.023 \mathrm{mM}$ inactivated the enzyme. Probe purification was completed by running $15 \mu \mathrm{L}$ of each in vitro transcription reaction on a $6 \%$ TBE-Urea polyacrylamide gel (Invitrogen, Carlsbad, CA, USA) at $180 \mathrm{~V}$ for approximately $1 \mathrm{~h}$. The probes were then excised from the gel and eluted from the excised gel plugs overnight at $37^{\circ} \mathrm{C}$ in SuperSignal ${ }^{\circledR}$ RPAIII ${ }^{\mathrm{TM}}$ Probe Elution Buffer (Pierce Biotechnology, Rockford, IL, USA). Gel purification is necessary when working with radioactive probes to eliminate unincorporated nucleotides that can cause smearing in the final RPA gel analysis. The purified radioactive probes were quantified by liquid scintillation counting.

\section{Preparation of Biotinylated Probes}

Probes biotinylated with Biotin-N44-CTP were prepared by in vitro transcription reactions using North 2 South $^{\mathrm{TM}} \mathrm{T} 7$ in vitro Transcription kits (Pierce Biotechnology) per the manufacturer's instructions and Ambion Antisense DNA templates. The in vitro transcription reaction was incubated at $37^{\circ} \mathrm{C}$ for $30 \mathrm{~min}$, and the excess original template digested with $2 \mathrm{U}$ DNase I for $15 \mathrm{~min}$ at $37^{\circ} \mathrm{C}$. Adding EDTA to a final concentration of $0.023 \mathrm{mM}$ inactivated the enzyme. Probes were precipitated with $7.5 \mathrm{M} \mathrm{LiCl}$ (final concentration $2.5 \mathrm{mM}$ ) at $-20^{\circ} \mathrm{C}$. The precipitated probes were collected by centrifugation at $16000 \times$ $g$ and washed once in ice-cold $70 \%$ ethanol. Gel purification of biotinylated probes is not necessary, since any unincorporated nucleotides remaining after DNase treatment are not retained on the nylon membrane during subsequent steps. The precipitated probes were resuspended in DEPC-treated water and quantified spectrophotometrically at $260 \mathrm{~nm}$.

\section{RPA and Detection Procedure}

RPA reactions and detection were performed using the Su-
perSignal RPAIII kit. Biotinylated probes $(80 \mathrm{pg})$ or radioactive probes $(10000 \mathrm{cpm} /$ approximately $15 \mathrm{pg})$ were hybridized to different amounts of total RNA (refer to specific figures). These probe levels yield a 4-fold molar excess of probe over $\beta$-actin mRNA target for an approximate $10 \mu \mathrm{g}$ normal tissue total RNA sample (E. Prediger, personal communication). This molar excess of probe is necessary for quantitative detection of mRNA. When probing for rare messages, the copy number is far less; therefore, the probe is in even greater molar excess. If more probe than this is used, it will only be degraded, potentially increasing the background (E. Prediger, personal communication).

Each probe/total RNA reaction was hybridized overnight at a temperature optimal for the specified probe composition in question (refer to figure legends for specified temperatures). After hybridization, each reaction was digested using a 1:100 or 1:25 dilution of RNase T1 (stock concentration of $5000 \mathrm{U} / \mathrm{mL}$ ) or RNase A/T1 (stock concentration of 250 $\mathrm{U} / \mathrm{mL}$ RNase A/10 $000 \mathrm{U} / \mathrm{mL}$ RNase T1) (refer to the specific figure) for $30 \mathrm{~min}$ at $37^{\circ} \mathrm{C}$. After RNase inactivation and RNA precipitation, the RNA pellet was resuspended in $8 \mu \mathrm{L}$ Gel Loading Buffer, vortex mixed, and heated at $95^{\circ} \mathrm{C}$ for 3 min. Entire digested reactions and a 10-fold dilution of undigested reactions were loaded onto a 6\% TBE-Urea gel (Invitrogen) and electrophoresed in $1 \times \mathrm{TBE}$ at $180 \mathrm{~V}$ for approximately $1 \mathrm{~h}$. For chemiluminescent detection, the gel was transferred to a Pierce Biodyne B positive nylon membrane in a Hoefer electroblotter containing $0.5 \times \mathrm{TBE}$ at $380 \mathrm{~mA}$ for 30 min. Transferred RNA was cross-linked to the membrane using an UV cross-linker equipped with 254-nm bulbs at 120 $\mathrm{mJ} / \mathrm{cm}^{2}$. Detection proceeded by incubating the blot in blocking buffer for 15 min on an orbital shaker, followed by incubation with stabilized streptavidin-HRP conjugate diluted 1:300 in blocking buffer for $15 \mathrm{~min}$. The membrane was washed $4 \times 5 \mathrm{~min}$ in wash buffer, washed $1 \times 5 \mathrm{~min}$ in substrate equilibration buffer, and incubated with the optimized detection reagents for $5 \mathrm{~min}$. Blots were covered with plastic wrap and exposed to X-ray film or to a FluorChem ${ }^{\mathrm{TM}} \mathrm{CCD}$ Camera (Alpha Innotech, San Leandro, CA, USA) for the times indicated in the figure legends.

For radioactive detection, the gel was dried on a gel drier at $80^{\circ} \mathrm{C}$ for 45 min using Whatman DEAE paper and exposed to X-ray film at room temperature overnight.

\section{RESULTS}

\section{Streptavidin/HRP-Based Chemiluminescent RPA Sensitivity Equals Radioactive RPA Sensitivity in a Fraction of the Time}

It was necessary for our chemiluminescent RPA to exhibit sensitivity at least equal to the traditional radioactive assay. The reason is clear: even with all of the practical advantages of chemiluminescence over radioactivity, if researchers had to compromise sensitivity, then there would be little advantage to a chemiluminescent alternative. Figure $1 \mathrm{~A}$ illustrates the results when a probe for $\beta$-actin, an abundantly transcribed message, is hybridized to decreasing amounts of total cellular RNA and detected using both chemiluminescent and radioactive methods. The limit of detection for both methods is approximately 60 ng total mouse liver RNA/80 pg $\beta$-actin probe. When exposure times are compared, however, a very significant difference 
is observed between the time it takes each method to detect this level of transcript. Chemiluminescent detection yielded very clear results with low background in a matter of minutes, whereas radioactivity required an overnight exposure.

When probing for rare transcripts such as c-myc, more target sequence is required ( $5 \mu \mathrm{g}$ vs. $60 \mathrm{ng}$ ), but a similar relationship in exposure times is observed (Figure 1B). The chemiluminescent RPA yields clear results with low background in minutes, while it takes an overnight exposure to achieve similar results when using radioactivity.

\section{Streptavidin/HRP-Based Chemiluminescent Detection System Exhibits Lower Background than Streptavidin/Alkaline Phosphatase-Based} Chemiluminescent Detection Systems

Chemiluminescent detection systems that utilize alkaline phosphatase conjugates also generate signal more quickly than the traditional radioactive methods. However, these alkaline phosphatase systems are commonly plagued with high backgrounds (7). Figure 2 illustrates the results when a rare message c-myc probe is hybridized to decreasing amounts of mouse thymus target total RNA and detected using a chemiluminescent alkaline phosphatase-based detection system. While the alkaline phosphatase system begins to generate specific signal in a similar amount of time as an HRP-based system, the background is significant and develops nearly as quickly as the probe signal. The low signal-to-noise ratio interferes with interpretation of the data, especially when detecting rare messages. In contrast, the HRP-based detection system provides the speed of a chemiluminescent detection system while minimizing background.

\section{Streptavidin/HRP-Based Chemiluminescent RPA Is Quantitative}

The linear response of the chemiluminescent RPA for an abundant message probe was determined by hybridizing serially diluted mouse liver total RNA to a constant amount of probe specific for the $\beta$-actin transcript and analyzing the signal of the protected fragments (similar to the experiment depicted in Figure 1A). The chemiluminescent signal was captured using a CCD camera with a linear range extending over almost 5 logs. This camera was used to calculate the density values corresponding to each band. Figure 3 illustrates that this new chemiluminescent RPA method is quantitative, yielding very linear results from a range of 30-1000 ng total RNA target present. The plot of concentration versus integrated optical density shows that the amount of $\beta$-actin message detected by chemiluminescence is proportional to the amount of total RNA added over a 30-fold range. A finite linear range of target cannot be defined for this assay because the response is different for every target depending on its level of expression. A linear response was observed with other targets as well (data not shown).

\section{Streptavidin/HRP-Based Chemiluminescent RPA Can Detect Multiple Transcripts in One Sample of Total RNA}

Since the hybridization step for an RPA is performed in solution, a key advantage of the RPA is the ability to probe simultaneously for multiple sequences in one complex sample of total cellular RNA. The success of this multiplexed assay hinges on careful probe design such that each probe length (both pre- and post-RNase digestion) is a unique size. Figure 4 shows how our new chemiluminescent RPA system enables

\section{A}
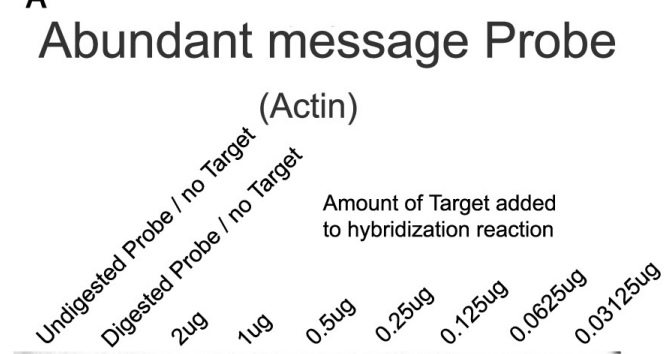

.

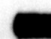

.

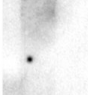

$\cdot$

Exposed for 6 minutes

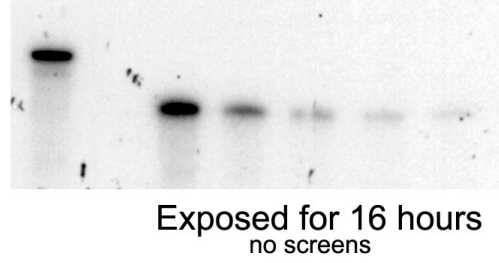

B

\section{Rare Message Probe}

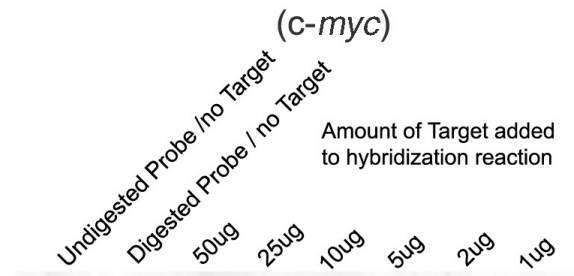

Chemiluminescence
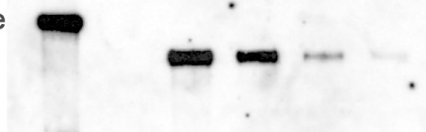

Exposed for 15 minutes

Radioactivity

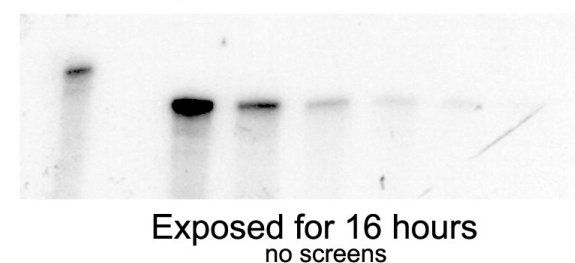

Figure 1. Comparison of chemiluminescent sensitivity to traditional radioactive RPA sensitivity with abundant and rare message probes. (A) RPA using the positive control system contained within the SuperSignal RPAIII kit. Lanes 1 and 2 were hybridized using $80 \mathrm{pg} / 10000 \mathrm{cpm} \beta$-actin probe and $2 \mu \mathrm{g}$ yeast torula RNA. Lanes 3-9 were hybridized using $80 \mathrm{pg} / 10000 \mathrm{cpm} \beta$-actin probe and the indicated amount of target (mouse liver total RNA). All reactions were hybridized overnight at $42^{\circ} \mathrm{C}$. Lanes $2-9$ were digested using a 1:100 dilution of RNase T1. (B) RPA probing for the rare c-myc transcript. Lanes 1 and 2 were hybridized using $100 \mathrm{pg} / 10000 \mathrm{cpm} \mathrm{c}-m y c$ probe and $50 \mu \mathrm{g}$ yeast torula RNA. Lanes 3-9 were hybridized using $100 \mathrm{pg} / 10000 \mathrm{cpm}$ of c-myc probe and the indicated amount of target (mouse thymus total RNA). All reactions were hybridized overnight at $56^{\circ} \mathrm{C}$. Lanes $2-9$ were digested using a $1: 25$ dilution of RNase T1. 
researchers to directly compare expression levels of c-myc, $\beta$ actin, Egr1, JunB, and cyclophilin in total RNA isolated from different tissues in a fraction of the time necessary for traditional radioactive methods. In Figure 4, different amounts of probe were used to optimize signal intensity. This approach enables comparisons of the target across different tissues. Alternatively, the specific activities of each probe can be adjusted to enable the comparison of targets within a tissue, as well as across different tissues. The levels of transcript seen in Figure 4 are predicted per customers that use radioactive RPAs who probe for the same multiple transcripts with the same antisense templates and tissues (E. Prediger, personal communication).

\section{Artifacts that Are Commonly Seen Using Biotinylated Probes in RPAs Can Be Eliminated}

We have discovered that the majority of biotinylated probes we examined that were specific for rare transcripts exhibited an artifact band at approximately 360 nucleotides after digestion with the recommended 1:100 dilution of RNase T1 or RNase A/T1 (6 out of 10 tested) using either streptavidin/alkaline phosphatase- or streptavidin/HRP-based systems. The same radiolabeled probes with identical assay conditions did not exhibit this artifact. Moreover, this artifact never appeared while examining biotinylated probes specific for abundant transcripts ( 0 out of 4 tested). The observed artifact at approx-
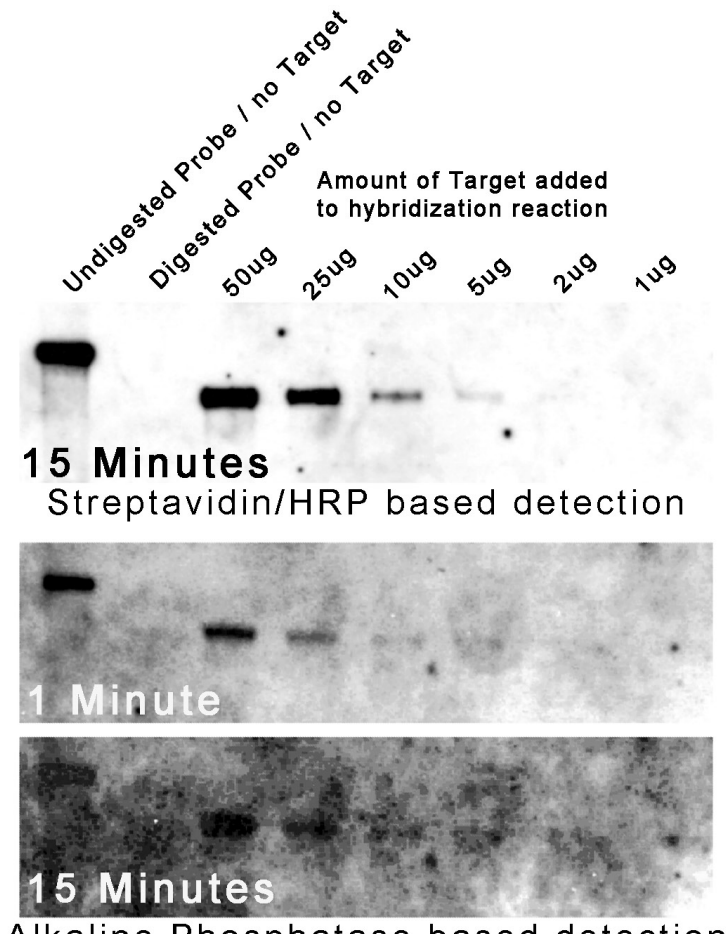

Alkaline Phosphatase based detection

Figure 2. Comparison of chemiluminescent detection: streptavidin/HRP versus streptavidin/alkaline phosphatase. Chemiluminescent RPAs probing for the rare c-myc transcript were performed and detected with either our streptavidin/HRP system or a streptavidin/alkaline phosphatase system. Lanes 1 and 2 were hybridized using $100 \mathrm{pg} / 10000 \mathrm{cpm} \mathrm{c}-m y c$ probe and $50 \mu \mathrm{g}$ yeast torula RNA. Lanes 3-9 were hybridized using $100 \mathrm{pg} / 10000 \mathrm{cpm}$ c-myc probe and the indicated amount of target (mouse thymus total RNA). All reactions were hybridized overnight at $56^{\circ} \mathrm{C}$. Lanes $2-9$ were digested using a 1:25 dilution of RNase T1. X-ray film exposure times are indicated in the respective panels. imately 360 nucleotides was independent of the specific ribonuclease used (data not shown), of the probe size, of the hybridization temperature (data not shown), and of the presence, absence, or abundance of target sequence (Figure 5A). Manipulating the concentration of RNase A or, more specifically, manipulating the concentration of the RNase A and T1 combination (Figure 5B) can eliminate this artifact. Digestion with RNase T1 exclusively is ineffective in removing the artifact.

While we have not yet identified the nature of the artifact, it is clearly a byproduct of exposure of the biotinylated RNA probe to RNase, yet is not eliminated by chloroform extraction. This implies that, while the ribonuclease was essential to its formation, the higher molecular weight artifact does not contain RNase or protein per se. Regardless of its composi-

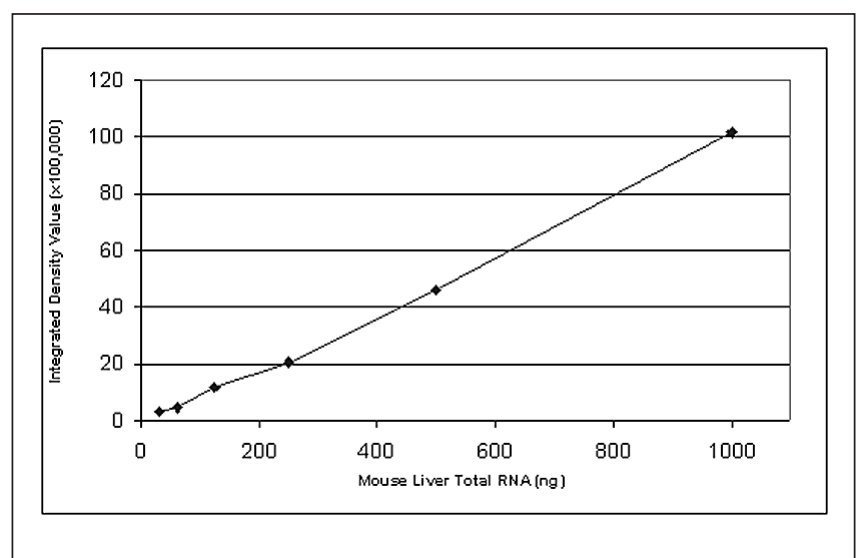

Figure 3. Linearity of the intensity of a $\boldsymbol{\beta}$-actin probe hybridizing to mouse liver total RNA. The integrated density value of the $31.25-1000 \mathrm{ng}$ bands in Figure 1A were measured on a CCD camera. Microsoft ${ }^{\circledR}$ Excel $^{\circledR}$ was used to graph these results against their corresponding RNA concentration and to determine a coefficient of correlation $\left(\mathrm{R}^{2}=0.996\right)$.

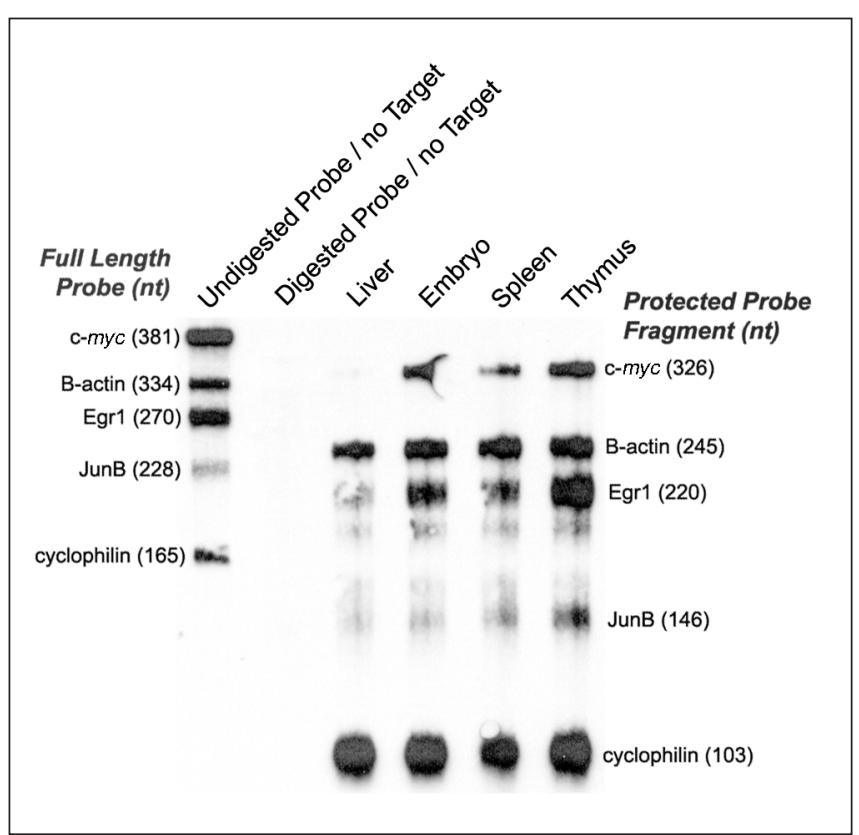

Figure 4. Multiple probe analysis of five transcript targets in four mouse tissue total RNAs. Chemiluminescent RPA using 100 pg c-myc, 10 pg $\beta$ actin, $50 \mathrm{pg}$ Egr1, $80 \mathrm{pg}$ JunB, $80 \mathrm{pg}$ cyclophilin, and $50 \mu \mathrm{g}$ each total mouse RNA. All reactions were hybridized overnight at $56^{\circ} \mathrm{C}$. Samples were digested with a 1:25 dilution of RNase A/T1. X-ray film exposure time was 2 min. nt, nucleotides. 
A

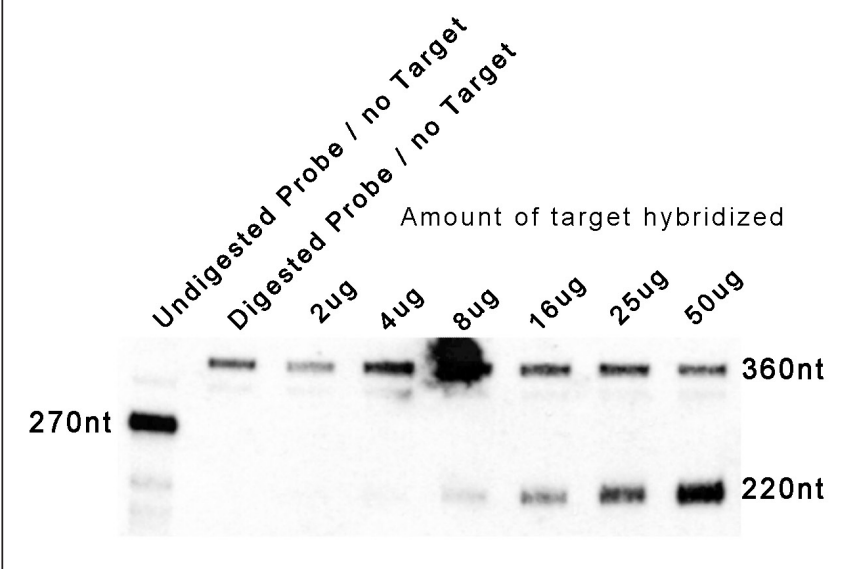

B

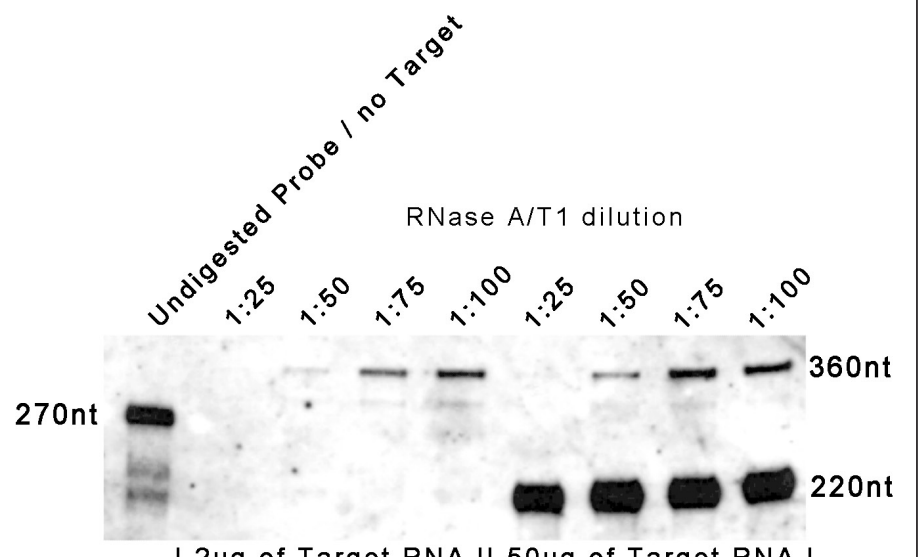

| 2 ug of Target RNA || 50 ug of Target RNA |

Figure 5. Characterization and elimination of an artifact commonly seen when performing RPAs using biotinylated probes. (A) Increasing amounts of total mouse thymus RNA were hybridized to $80 \mathrm{pg}$ Egr1 probe and detected with our streptavidin/HRP-based RPA. All samples were hybridized overnight at $42^{\circ} \mathrm{C}$. Note the presence of the artifact in the digested probe/no target negative control lane. Samples were digested with a 1:100 dilution of RNase T1. Exposure time was $60 \mathrm{~min}$. (B) Increasing amounts of RNase A/T1 were added to reactions containing $80 \mathrm{pg}$ Egr 1 probe hybridized to either 2 or $50 \mu \mathrm{g}$ total mouse thymus RNA and detected with our streptavidin/HRP-based RPA. X-ray film exposure time was $60 \mathrm{~min}$.

tion, this artifact appears as a clear band independent of target concentration and could therefore be easily confused with the probe signal.

\section{DISCUSSION}

The RPA is an effective way to measure mRNA transcripts in complex mixtures of total RNA and is therefore a valuable tool in monitoring protein regulation and gene expression. We have developed a chemiluminescent streptavidin/HRP-based RPA that maintains the advantages that distinguish RPAs over other mRNA detection methods such as Northern blotting and RT-PCR. Our chemiluminescent RPA also improves on previous nonradioactive RPAs (6) by combining RNase inactivation and precipitation steps, as well as utilizing enhanced detection reagents. These advances significantly decrease assay time while improving sensitivity. As with all RPAs, hybridizing in solution gives the researcher the ability to use a large amount of target, enabling easier detection of rare messages, as well as to detect multiple transcripts in one sample of cellular total RNA. This chemiluminescent RPA alternative exhibits sensitivity comparable to radioactivity for both rare and abundant message probes. This sensitivity level is achieved in less than $1 \mathrm{~h}$ (frequently less than $10 \mathrm{~min}$ ), as opposed to radioactive results, which take many hours (often overnight) to visualize, and without background problems common to chemiluminescent alkaline phosphatase-based systems.

The advent of rapid hybridization solutions (3) that incorporate novel hybridization buffers for use with RPAs is another possible way to make this chemiluminescent RPA even more efficient. These solutions commonly decrease hybridization times from days or hours to minutes. Although we have not investigated these reagents, they could further expedite our RPA method by enabling a researcher to potentially generate RPA results without overnight hybridization.

\section{ACKNOWLEDGMENTS}

The authors thank other members of our laboratory, as well as David Brown and Ellen Prediger (Ambion) for their help, especially interpreting results concerning the artifact commonly seen at 360 nucleotides when performing RPAs using rare transcript biotinylated probes.

\section{REFERENCES}

1.Anderson, L. and A. Seilhamer. 1997. A comparison of selected mRNA and protein abundances in human liver. Electrophoresis 18:533-537.

2.Frayn, K.N., D. Langin, C. Holm, and P. Belfrage. 1993. Hormone-sensitive lipase: quantitation of enzyme activity and mRNA level in small biopsies of human adipose tissue. Clin. Chim. Acta 216:183-189.

3.Harthoorn, L.F., R.C. Oudejans, J.H. Diederen, D.J. Van de Wijngaart, and D.J. Van Horst. 2001. Absence of coupling between release and biosynthesis of peptide hormones in insect neuroendocrine cells. Eur. J. Cell Biol. 80:451-457.

4.Hobbs, M.V., W.O. Weigle, D.J. Noonan, B.E. Torbett, R.J. McEvilly, R.J. Koch, G.J. Cardenas, and D.N. Ernst. 1993. Patterns of cytokine gene expression by CD4+ T cells from young and old mice. J. Immunol. 150:3602-3614.

5.Lee, J.J. and N.A. Costlow. 1987. A molecular titration assay to measure transcript prevalence levels. Methods Enzymol. 152:633-648.

6.Nass, S.J. and R.B. Dickson. 1995. Detection of cyclin messenger RNAs by nonradioactive ribonuclease protection assay: a comparison of four detection methods. BioTechniques 19:772-778.

7.Turnbow, M.A. and C.W. Garner. 1993. Ribonuclease protection assay: use of biotinylated probes for the detection of two messenger RNAs. BioTechniques 15:267-270.

8.Zinn, K., D. DiMaio, and T. Maniatis. 1983. Identification of two distinct regulatory regions adjacent to the human $\beta$-interferon gene. Cell 34:865-879.

Address correspondence to Christopher Rosenau, Pierce Milwaukee, LLC, 2202 N. Bartlett Avenue, Milwaukee, WI 53202,USA.e-mail: christopher.rosenau@piercenet.com 